\title{
Non-Arteritic Anterior Ischemic Optic Neuropathy and Factor V Leiden
}

\section{Lorca-Barchín J, Medrano-Martínez V $\mathrm{V}^{*}$, Francés-Pont I, Fernández-Izquierdo S, Mallada-Frechin J and Piqueras-Rodríguez L}

\author{
Neurology Department, Hospital general universitario virgen de la salud, Elda, Spain
}

*Corresponding author: Medrano-Martínez V, Neurology Department, Hospital general universitario virgen de la salud, Carretera elda-sax, s/n. Elda/Alicante, Cp: 03600, Spain, E-mail: vmedrano714k@icloud.com

Citation: Lorca-Barchín J, Medrano-Martínez V, Francés-Pont I, Fernández-Izquierdo S, Mallada-Frechin J, et al. (2015) Non-Arteritic Anterior Ischemic Optic Neuropathy and Factor V Leiden. J Neurol Neurol Disord 2(3): 303.

Received Date: September 03, 2015 Accepted Date: September 29, 2015 Published Date: September 30, 2015

\begin{abstract}
Non-arteritic anterior ischemic optic neuropathy (NAION) is an entity that is caused by circulatory insufficiency which affects the proximal portion of the optic nerve. NAION is characterized by the following triad: abrupt and painless loss of vision, optic disc smelling and nerve fiber bundle defects in the visual field of the affected eye. Cardiovascular risk factors and hrombophilic disorders have been proposed in the pathogenesis of NAION, such as Factor V Leiden thrombophilia (FVL). Factor V Leiden is a inherited disorder of blood clotting that predisposes to venous thrombotic events. Here, we report the case of 44-year-old female patient with NAION and activated protein C resistente (or FVL) who visited our hospital, and the diagnosis is discussed. We also review the literature of these two conditions. We suggest that physicians consider prothrombotic disorders when evaluating patients with AION, especially in atypical cases or cases occurring in young patients without classic factors of cardiovascular risk.
\end{abstract}

Keywords: NAION; Ocular vascular occlusion; Hypercoagulability disorders; Factor V Leiden;

\section{Introduction}

Non-arteritic anterior ischemic optic neuropathy (NAION) is an ischemic condition which affects the prelaminar portion of the optic nerve head. That is supplied by the ciliary arteries, and central retinal artery [1]. Yet, meaningful progress toward understanding the pathogenesis of this entity has been limited [2]. Thrombophilic disorders are proposed in the pathogenesis of NAION, such as resistance to activated protein C (APC resistance), protein C or S deficiency, factor V Leiden (FVL) and hyperfibrinogenemia. However, when the name of an entity itself may be a misnomer, it can be especially difficult to escape erroneous interpretations of pathophysiology. Indeed, the mechanism of this disease needs to be reanalyzed in a completely different direction. We present a case of NAION associated with FVL that we attended in our hospital and we review the literature of these two entities [2].

\section{Case Report}

Female, 44 years, who visited the Emergency Service referring blurred vision in the lower hemifield of the left eye with $48 \mathrm{~h}$ of evolution. The patient did not exhibit relevant ophthalmological and others antecedents. Not coexist cardiovascular risk factors but usually the patient takes oral contraceptives pills since 4 years. Ophthalmological examination revealed a visual acuity of 1.2 in both eyes and intraocular pressure within normal range. Biomicroscopy didnot show significant alterations, with relative afferent pupil defect and without pain with extraocular movements. Relevant findings of funduscopy were a unilateral papillary sectorial edema and oclusión arteriolar lower hemifield of the lefg eye. Campimetry (OCTOPUS 1-2-3, Stimuli 3), (Figure 1). Encephalic resonance and ocular resonance did not determine significant alterations. Urgent analysis was requested which did not exhibit alterations in basic hemogram study, coagulation or biochemistry, including acute phase reactants. Subsequently, analytics screening for infections was performed and serology for Borrelia burgdorferi, Rickettsia conorii, human immunodeficiency virus, chickenpox and syphilis was requested, and all were negative. Although hypercoagulability don't show significant alterations (antipospholipid antibodies determination, protein $\mathrm{C}$ or $\mathrm{S}$ deficiency, hyperfibrinogenemia, hyperhomocysteinemia, lupus anticoagulant, antithrombin III deficiency was all negative), patient found to be heterozygous for the coagulation factor V (F5 $1691 \mathrm{G}>\mathrm{A}$ [Arg>Gln rs6025/560]) risk allele that predisposes one to a genetically inherited disorder of blood clotting, Therapy was initiated with aspirine $100 \mathrm{mg}$ b.d, and oral contraceptive pills was stoped. After one year of follow-up the patient did not exhibit new episodes, with reduction but persistence of inferior altitudinal defect in campimetry. Control of retinal angiography was normal (Figure 2). 

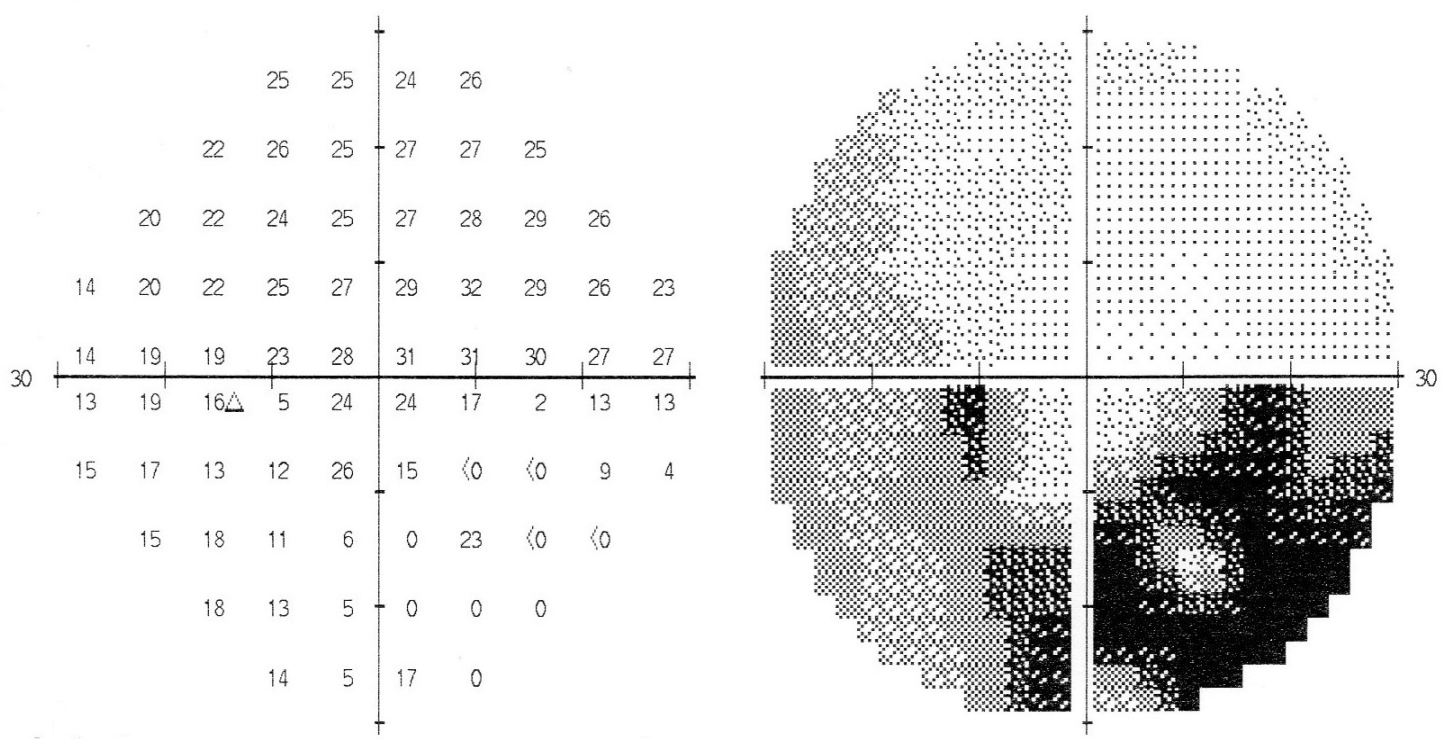

Figure 1: Relevant findings of campimetry were a unilateral papillary sectorial edema and oclusión arteriolar lower hemifield of the lefg eye. Hospital General Universitario Virgen de la Salud de Elda, Spain

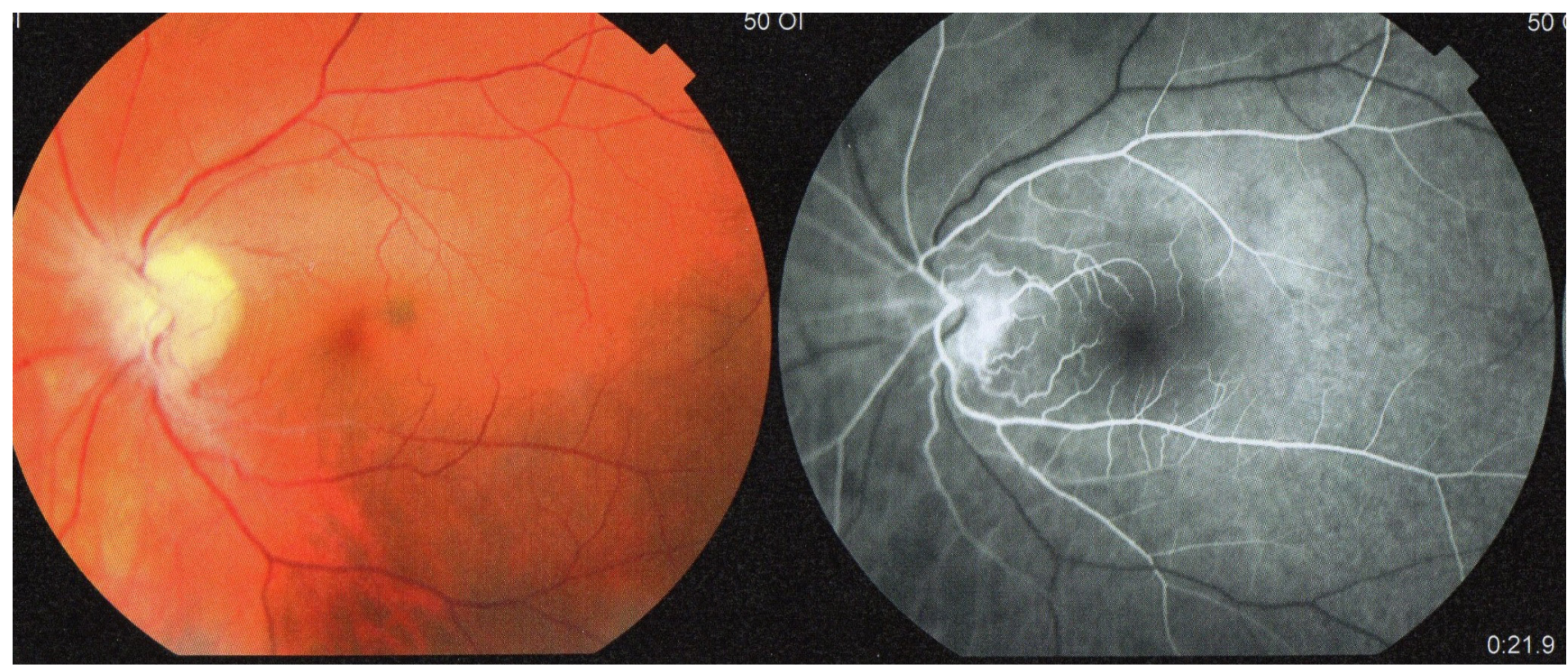

Figure 2: Normal control of retinal angiography. Hospital General Universitario Virgen de la Salud de Elda, Spain

\section{Discussion}

Non-arteritic anterior ischemic optic neurophaty is the most common acute optic neuropathy after age 50. It is presumed to result from circulatory insufficiency within the optic nerve head. NAION occurs between the ages of 45 and 65 , usually over 50 years [3], and it is not attributable to inflammation, demyelination disease, metastatic infiltration, mass effect or orbital congestion. It is characterized by painless monocular visual loss, altitudinal visual fields defects and afferent pupillary defect (or Marcus Gunn pupil), associated with optic disc swelling of a pallid nature, sometimes with flame hemorrhages on the swollen disc. Association of NAION with cardiovascular risk factors in adults -hypertension, nocturnal hypotension, diabetes mellitus, hyperlipidemia, smoking, ischemic heart disease, among others-is not firmly documented [3,4]. However, there have been reported NAION cases in patients with hypercoagulable states: the so-called thrombophilic factors, such as factor V Leiden thrombophilia [5] (FVL). Such mutations may actually be found in over 6\% of the European population and has not been shown to be associated with NAION in a significant manner. Hypercoagulability does contribute the pathogenesis of the disease, then perhaps the contribution of the patients oral contraceptive pills should be further discussed, as this highly increases the risk for venous thrombosis, especially in combination with FVL.

The FVL is a mutation of one of the clotting factors in the blood called factor V that results in thrombophilia, which is an increased tendency to form abnormal blood clots that can block blood vessels. Other factors involved in thrombophilia are protein $\mathrm{C}$ or S deficiency, hyperfibrinogenemia, hyperhomocysteinemia, lupus anticoagulant, antithrombin III deficiency, among others. Sometimes several factors coexist with each other or with cardiovascular risk factors and increased incidence of NAION (synergi- 
stic effect), such as the case reported by Srinivasan, et al. [6] of a patient with primary antiphospholipid syndrome and FVL. A case of NAION in a patient with PAI-1 4G/5G mutation, FVL and hyperlipidemia was also reported by Titlic, et al. [7]. Glueck, et al. [8] reported a case of a family with concomitant FVL and prothrombin 20210 mutation that had a broad spectrum of thrombotic phenomena: NAION or amaurosis fugax, deep vein thrombosis, hemispheric ischemic stroke, transient ischemic attack, mesenteric arterial thrombosis, among others.

Some authors have developed retrospective, analytical studies to determine association between hypercoagulable disorders and NAION, such as Nagy, et al. [9]. This case-control study found that $24 \%$ of patients with NAION had resistance to APC compared to $5.9 \%$ in control group, therefore, it show that patients with factor V Leiden had a significantly increased risk of developing NAION $(\mathrm{p}<0.021)$. In 2006, Nagy, et al. published another case-control study in which factor V Leiden, several thrombophilic conditions and cardiovascular risk factors was determined. In this case-control, lipoprotein A (OR 16.88), factor V Leiden (OR 5.78), diabetes mellitus (OD 4.44), levels of von Willebrand factor, hypercholesterolemia and hyperfibrinogenemia proved significant risk factors for NAIOIN $(\mathrm{p}<0.05)$ [10]. Recently in 2015 Schockman, et al. [11], reported a study with 265 patients with ocular vascular occlusion (vein and artery occlusion). Patients were sequentially referred from vitreoretinal specialists for measurement of thrombophilia-hypofibrinolysis and compared to healthy normal controls. Of the 265 patients with ocular vascular occlusion, (11\%) had factor V Leiden and/or prothrombin gene, and $83 \%$ of these 29 cases presented with ocular vascular occlusion as their first thrombotic event.

Diagnosis of NAION should be mainly based on clinical data on physical examination and exclusion of potential causes. Confirmation of causal relation between hypercoagulable state and NAION should facilitate its diagnosis and treatment as well as preventing thrombotic events in other vascular territories. Thrombophilia testing is not recommended in a systematic way, but in cases with atypical presentation, for example, patients with a personal or family history that suggest a predisposition to thrombogenesis, or young patients without classic factors of cardiovascular risk.

\section{References}

1. Miller NR, Arnold AC (2015) Current concepts in the diagnosis, pathogenesis and management of nonarteritic anterior ischaemic optic neuropathy. Eye 29: 65-79.

2. Parsa CF, Hoyt WF (2015) Nonarteritic anterior ischemic optic neuropathy (NAION): a misnomer. Rearranging pieces of a puzzle to reveal a nonischemic papillopathy caused by vitreous separation. Ophthalmology 122: 439-42.

3. Rucker JC, Biousse V, Newman NJ (2004) Ischemic optic neuropathies. Curr Opin Neurol 17: 27-35.

4. Buono LM, Foroozan R, Sergott RC, Savino PJ (2002) Nonarteritic anterior ischemic optic neuropathy. Curr Opin Ophthalmol 13: 357-61.

5. Worrall BB, Moazami G, Odel JG, Behrens MM (1997) Anterior ischemic optic neuropathy and activated protein C resistance. A case report and review of the literature. J Neuroophthalmol 17: 162-5.

6. Srinivasan S, Fern A, Watson WH, McColl MD (2001) Reversal of nonarteritic anterior ischemic optic neuropathy associated with coexisting primary antiphospholipid syndrome and Factor V Leiden mutation. Am J Ophthalmol 131: 671-3.

7. Titlic M, Karaman K, Andelinovic S (2009) Anterior ischemic optic neuropathy comorbid with Factor V Leiden and PAI-1 4G/5G mutation. Bratisl Lek Listy 110: $192-4$.

8. Glueck CJ, Wang P (2009) Ocular vascular thrombotic events: a diagnostic window to familial thrombophilia (compound factor V Leiden and prothrombin gene heterozygosity) and thrombosis. Clin Appl Thromb Hemost 15: 12-8.

9. Nagy V, Facsko A, Takacs L, Balazs E, Berta A, et al. (2004) Activated protein C resistance in anterior ischaemic optic neuropathy. Acta Ophthalmol Scand 82: $140-3$.

10. Nagy V, Steiber Z, Takacs L, Vereb G, Berta A, et al. (2006) Trombophilic screening for nonarteritic anterior ischemic optic neuropathy. Graefes Arch Clin Exp Ophthalmol 244: 3-8.

11. Schockam S, Glueck CJ, Hutchins RK, Patel J, Shah P, et al. (2015) Diagnostic ramifications of ocular vascular occlusion as a first thrombotic event associated with factor V Leiden and prothrombin gene heterozygosity. Clin Ophthalmol 9: 591-600.

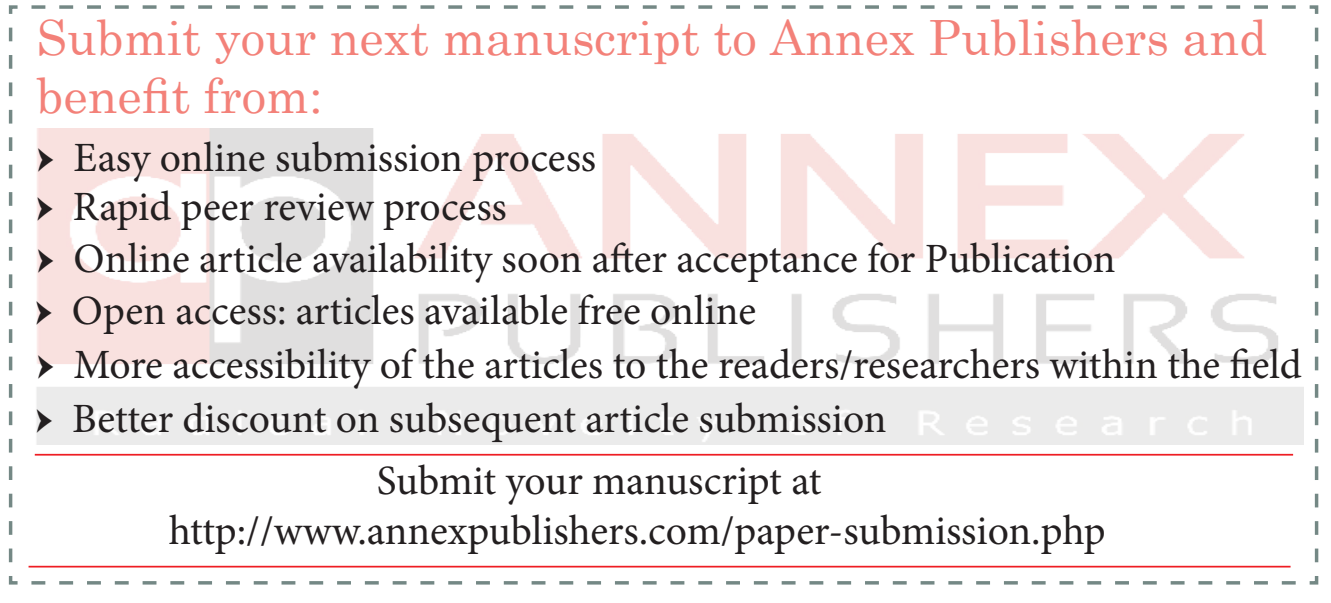

